\title{
Clinical and Laboratory Parameters Associated with Acute Kidney Injury in Viper Envenomed Cases
}

\author{
Hoda M Salah Eldin and Rabab Nabil Hafez ${ }^{1}$
}

${ }^{1}$ Department of Forensic Medicine \& Clinical Toxicology, Faculty of Medicine, Ain Shams University, Cairo, Egypt.

\begin{abstract}
Viper species are distributed allover Egypt. Venomous bites by Viperidae snakes are associated with local and systemic manifestations and may be exacerbated by the development of acute kidney injury (AKI).

THE AIM of the study was to assess acute renal injury in cases of envenomations by locally prevalent vipers as regards incidence, clinical and laboratory parameters and final outcome.

METHODS: The current study was conducted on all patients, of both sex, admitted in Poison Control Center of Ain Shams University hospitals following envenomation by Viperidae snakes during 2014, 2015, and 2016. Diagnosis was based on history of snake bite with suggestive clinical data. Recorded data included patient characteristics (age, gender, delay time), vital data (heart rate, blood pressure), presence of local signs, bleeding manifestations, laboratory parameters (INR, platelet count, hemoglobin level, BUN and serum creatinine), number of antivenom vials used , duration of hospital stay and survival. Cases were sub-grouped to group I with AKI and group II without AKI.

RESULTS: A total of 120 cases of venomous bites by Viperidae snakes were recorded during the study period with mortality of $4.2 \%$. Cases were predominantly males with mean age of $35+/$ 14.7years. A subset of 5.8\% cases developed AKI which statistically was related to older age, longer delay time, increased incidence of bleeding manifestations and tachycardia, higher values of INR, serum creatinine, and blood urea nitrogen, lower platelet count, higher number of used antivenom vials and longer duration of hospital stay. Gender, hypotension, presence of local manifestations and hemoglobin level were insignificant among AKI. Mortality among AKI cases was statistically linked to old age, prolonged delay time, increased incidence of bleeding manifestations, higher INR values, low platelet count and low hemoglobin level and increased number of used antivenom vials and duration of hospital stay. The incidence of tachycardia, hypotension and local manifestations, serum creatinine and blood urea nitrogen levels were not statistically different among non- survivors.

CONCLUSION: Acute renal injury may complicate the course of viper envenomations. Venom hemotoxicity is related to morbidity and mortality especially with older age and longer time between bite to antivenom therapy.

Bisphenol A, Vitamin E, Testis, Prostate, Spermatogenesis.
\end{abstract}

Keywords acute kidney injury, viper envenomation, Egypt

\section{Introduction}

$\mathrm{S}$ nakebite is a common medical emergency worldwide. In Africa, official snakebite statistics is inadequate and fragmentary with difficulty in accurate assessment of snakebite-related morbidity and mortality. Preliminary estimates of 3-8 thousands envenomations occur in North Africa and Middle East, although far fewer mortalities are reported each year. Despite the presence of a number of venomous snake families in Africa, envenomation by snakes of the family Viperidae alone cause approximately $60 \%$ of all bites (Kasturiratne et al., 2008; World Health Organization, 2010).

Several species of vipers and cobras with the ability to deliver deadly venom are found in Egypt.
Snakes in Egypt are found in a wide variety of habitats ranging from the vast sandy deserts to the fertile Nile delta. The common viper genera found in Egypt are Cerastes, Psuedocerastes, and Echis (Baha, 2006; Bernardoni et al., 2014).

Viper envenomation is characterized by rapidly developing prominent local effects, including necrosis, hemorrhage, edema, and pain at the bite site, which my result in permanent sequelae. This is in addition to systemic alterations such as hemorrhage, coagulopathy, shock, and acute renal failure (Mandal, 2015).

The kidney, as a highly vascularized organ with excretory function, is prone to venom toxicity. 
Renal involvement in viper envenomations has received interest by researchers in the tropics especially in Asia and South America. However, the great diversity in snake population and venom constituents from species to species and within the same species according to geographic region, differences in study populations, accessibility of medical service and study designs all leads to variability of clinical effects across studies (Athappan et al.,2008 ; Kasturiratne et al., 2008 ; Kanjanabuch and Sitprija,2008; Potdukhe et al.,2017).

The current study was conducted in Poison Control Center of Ain Shams University hospitals (PCC-AUH) aiming at assessment of acute kidney injury (AKI) in cases of envenomations by locally prevalent vipers as regards incidence, clinical and laboratory parameters and outcome .

\section{Subjects \& Method}

The present study was planned as a hospital-based retrospective observational study.

\section{Study group}

All patients, of both sex, who were admitted in PCCAUH following envenomation by snakes of the family Viperidae during 2014, 2015, and 2016 were enrolled in this study. Diagnosis was based on history of snake bite with identification of a clinical picture suggestive of snake envenomation by Viperidae which was defined as presence of fang marks, bleeding tendencies with local swelling, erythema, cellulites and abnormal clotting time (World Health Organization, 2010; World Health Organization, 2016).

Exclusion criteria: history of co-morbid illnesses including diabetes mellitus, cardiovascular diseases, renal diseases, liver diseases, and hematologic diseases or concomitant use of drugs.

Data:

The current study was approved by both the general director of PCC-AUH and the Research Ethics Committee of Faculty of Medicine Ain Shams University. Anonymised data were collected from medical records with consideration of confidentiality issues. Recorded data were tabulated and statistically analyzed using standard SPSS (Standard Package for Social Science) software package, version 20 (Chicago. IL).

Cases were sub-grouped according to renal impairment into:

- Group I: included cases with acute kidney injury (AKI) as defined by an increase in serum creatinine $\geq 0.3 \mathrm{mg} / \mathrm{dL}(\geq 26.5 \mu \mathrm{mol} / \mathrm{L})$ within $48 \mathrm{~h}$ according to the Acute Kidney injury Network (AKIN) classification (Lopez \& Jorge, 2013).

- Group II: Cases without AKI as defined by BUN and serum creatinine within normal.

Data in the present study included:

1. Patient characteristics:

- Age, Gender, Delay time

2. Vital data: including

- Heart rate: Normal heart rate was considered between 60-100 beats/minute (Kenney et al., 2015).
- Blood pressure: Hypotension was considered if the patient's systolic blood pressure reading was lower than $90 \mathrm{mmHg}$ and diastolic blood pressure reading was lower than $60 \mathrm{mmHg}$, while hypertension was considered if the patient's blood pressure was more than 140/90 mmHg. (Kotchen, 2015).

3. Clinical manifestations of envenomation (local and systemic) :were defined according to the WHO 2016 guidelines for the management of snakebites (World Health Organization, 2016)

- Local such as fang marks, local swelling, erythema, cellulitis, regional lymphadenopathy

- Hematologic signs:

a) Clinically significant bleeding tendencies

- Locally: e.g. prolonged bleeding from fang marks or venipuncture

- Systemically: manifested by skin bleeding (including petechiae, purpura, and/or ecchymosis), mucosal bleeding (including gum bleeding, hematemesis, melena, hematochezia, gross hematuria, and/or vaginal bleeding)

4. Laboratory parameters:

a) INR (international normalized ratio) (seconds)

b) Platelet count $\left(10^{3} / \mathrm{mm}^{3}\right)$

c) Hemoglobin level (g/dl)

d) Renal function tests:

- Blood urea nitrogen(mg/dl)

- Serum creatinine(mg/dl)

5. Antivenom therapy:

Polyvalent lyophilized anti- snake serum vials produced by VACSERA, Egypt were used. Doses were according to manufacturer's recommendations and treatment protocols followed by PCC, starting by an initial dose of 5-10 vials by slow intravenous infusion after dilution with normal saline at a rate of $5-10 \mathrm{ml} / \mathrm{kg}$ body weight over $1 \mathrm{~h}$. Then this is followed by further dose of five vials if signs and symptoms of toxicity persist until improvement of symptoms (VACSERA, 2002)

6. Duration of hospital stay.

7. Survival.

\section{Results}

A total of 120 cases were admitted and reported as venomous bites by Viperidae snakes in PCC-ASUH between January 2014 and December 2016 with case mortality of $4.2 \%$. Distribution of cases and mortality during the years of the study is shown in table (1). Regarding age and gender distribution, mean age of cases was 35+/-14.7years with males constituting $82.5 \%(n=99)$ and females $17.5 \%(n=21)$

Cases with AKI (group I) represented 5.8\% $(n=7)$ of total cases. Case fatalities recorded in the three years of the study were all among group I cases, hence they were further classified according to survival into:

$\checkmark$ Survivors: (n=2).

$\checkmark$ Non-survivors: $(\mathrm{n}=5)$. 
Comparison between cases group I and group II:

There was a statistically significant higher mean age, and longer mean delay time among group I cases, while no significant difference between both groups regarding gender. Regarding clinical parameters and investigations, the occurrence of bleeding manifestations and tachycardia was significantly higher among group I. Additionally, group I showed statistically significant higher mean values of INR, serum creatinine, blood urea nitrogen, and lower mean platelet count. On the other hand, there was insignificant difference regarding incidence of hypotension, presence of local manifestations and hemoglobin level between both groups. Mean number of used antivenom vials and duration of hospital stay were significantly higher among group I in comparison to group II (Table2).

Comparison among group I cases according to survival:

Means values for age and delay time were significantly increased among non -survivors. Nonsurvivors showed significantly increased incidence of bleeding manifestations, higher INR mean values, lower platelet count and lower hemoglobin level. Conversely, the incidence of tachycardia, hypotension and local manifestations, in addition to mean creatinine and blood urea nitrogen levels were not statistically different compared to survivors. Increased mean number of used antivenom vials and duration of hospital stay were noted among non-survivors (Table3).

Table (1): Distribution of snake envenomed cases and mortality rates in the years 2014, 2015 and 2016.

\begin{tabular}{|c|c|c|c|c|c|}
\hline \multirow{2}{*}{ Year } & \multirow{2}{*}{$\begin{array}{c}\text { number of } \\
\text { cases }\end{array}$} & \multicolumn{2}{|c|}{ Survival } & \multicolumn{2}{|c|}{ mortality } \\
\cline { 3 - 6 } & 38 & 37 & $97.4 \%$ & $\mathbf{n}$ & $\mathbf{\%}$ \\
\hline $\mathbf{2 0 1 4}$ & 42 & 39 & $92.9 \%$ & 3 & $2.6 \%$ \\
\hline $\mathbf{2 0 1 5}$ & 40 & 38 & $95 \%$ & 2 & $5.1 \%$ \\
\hline $\mathbf{2 0 1 6}$ & 120 & 115 & $95.8 \%$ & 5 & $4.2 \%$ \\
\hline
\end{tabular}

n: Number of patients

Table (2): Statistical analysis showing comparison between group I and group II cases as regards patient characteristics, vital data, clinical manifestations of envenomation, laboratory parameters, antivenom therapy and duration of hospital stay.

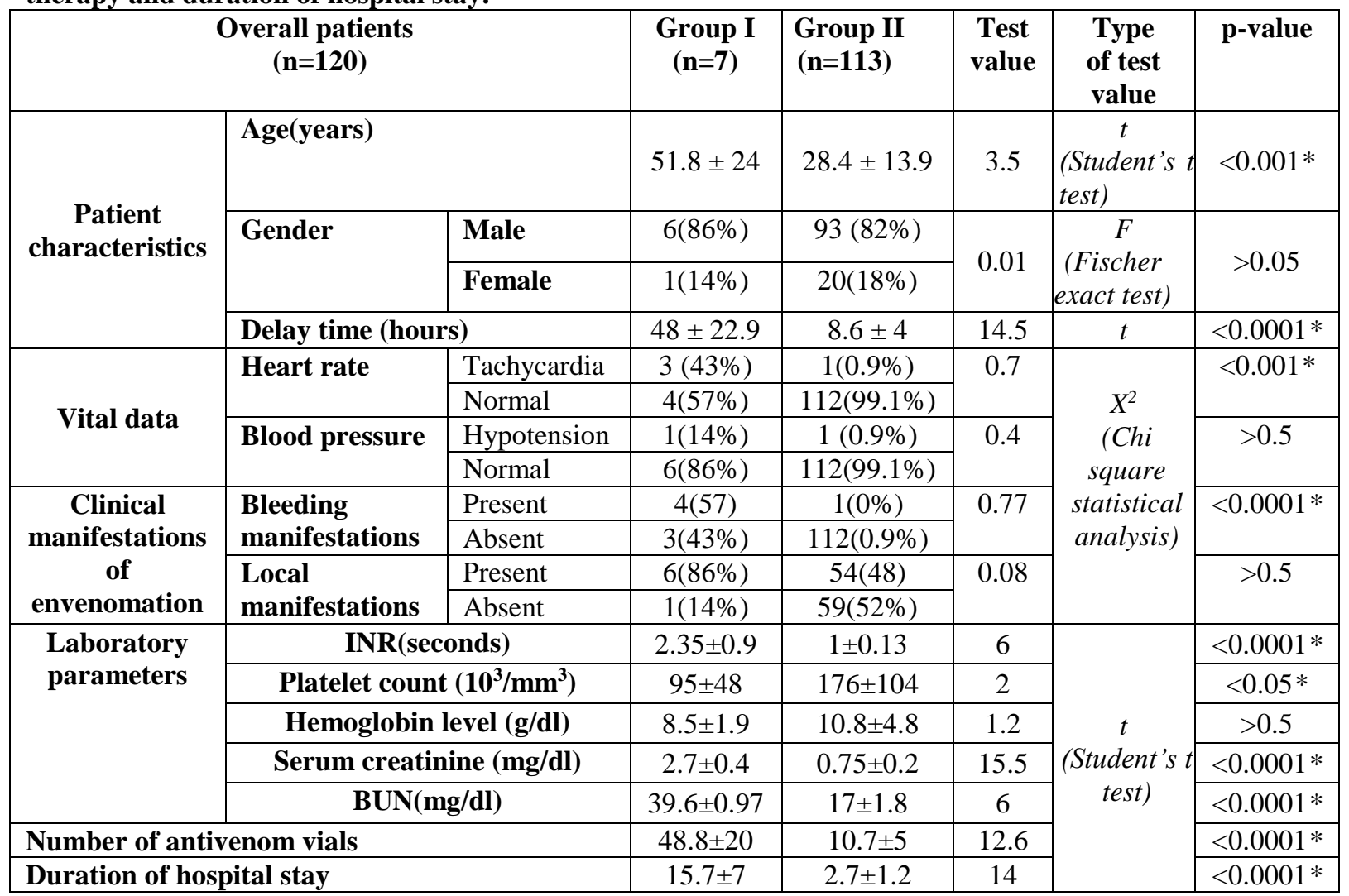

Data are presented as mean \pm standard deviation unless otherwise indicated, $n:$ Number of patients

INR: International normalized ratio, BUN: Blood urea nitrogen, $* P<0.05$ : statistically significant, $p>0.05=$ statistically non-significant 
Table (3): Statistical analysis showing comparison between survivors and non-survivors in group I cases as regards patient characteristics, vital data, clinical manifestations of envenomation, laboratory parameters, antivenom therapy and duration of hospital stay.

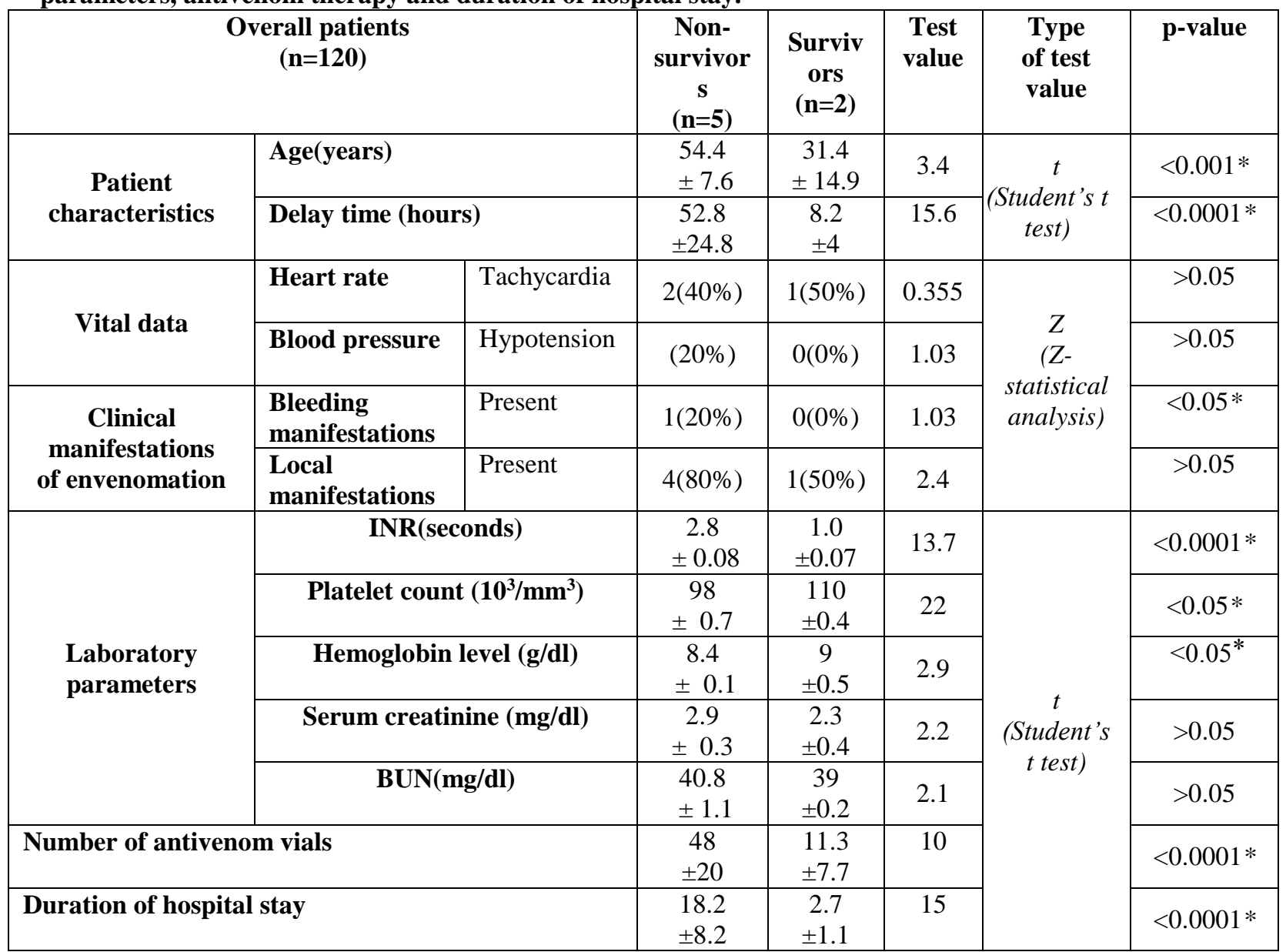

Data are presented as mean \pm standard deviation unless otherwise indicated, $n:$ Number of patients

INR: International normalized ratio, BUN: Blood urea nitrogen, ${ }^{*} P<0.05$ : statistically significant, $p>0.05=$ statistically non-significant

\section{Discussion}

Snake envenomation is a major problem in South Africa and Middle East however, there remains limited information of the clinical effects.The present study evaluated the incidence, clinical predictors and outcome of acute renal impairment which may complicate the course of viper envenomations among cases who were admitted in (PCC-AUH) during a three years period.

The incidence of acute renal injury in the current study was $5.8 \%$.Comparable results were recorded in case series in Nigeria and Thailand, while the incidence reported across Asia especially Sri Lanka and India was much higher. Being less common in Egypt, the non-recognition of acute renal impairment as a possible complication of viper envenomation could lead to delayed medical care and poor outcome (Kanjanabuch \& Sitprija, 2008; Harshavardhan et al., 2013).

In the current study, though victims of viper envenomations were predominantly males owing to common involvement in outdoor activities, there was no significant association of gender with occurrence of AKI after envenomation. Results suggested that older age was not only related to the occurrence of AKI but also to mortality. The increased incidence in older age was similarly noted by Athappan et al. (2008) and Singh et al. (2016).The predisposition of older kidney to acute kidney injury can be related to agerelated progressive structural and functional deterioration. These change include decline in glomerular filtration rate and renal blood flow in addition to altered response to vasoactive stimuli. These alterations occur jointly with structural changes namely loss of renal mass, hyalinization of afferent arterioles, and development of aglomerular arterioles, sclerotic glomeruli and tubule-interstitial fibrosis (Weinstein \& Anderson, 2010).

Findings in the present study showed that prolonged delay time to hospital had a significant relationship with both development of AKI and subsequent mortality. A number of authors noted the association of increased morbidity and mortality in subjects presenting late after viper 
bites. This finding reflects the delay in administration of antivenom with continuous damaging effects of the un-neutralized venom (Osmani et al., 2007; Athappan et al., 2008; Dharod et al., 2013).

Regarding clinical parameters, increased serum creatinine and blood urea nitrogen during hospital stay was used as an indicator of AKI, however levels were not statistically related to mortality.

Acute kidney injury (formerly known as acute renal failure) is a syndrome characterized by the rapid loss of the kidney's excretory function and is typically diagnosed by the accumulation of end products of nitrogen metabolism (urea and creatinine) or decreased urine output, or both. Rapid rises in blood urea nitrogen and serum creatinine occur due to the catabolic nature of the disease (Bellomo et al., 2012).

Various authors previously reported that acute renal impairment can develop early after envenomation by vipers and antivenom administration may be incapable to reverse the damage which have been already done. Renal failure may constitute an evidence of the earlier effects of venom and does not necessarily imply that the victim currently has un-neutralized venom in the system (Isbister et al, 2009; Manafa et al, 2016; Potdukhe et al., 2017)

Among cases with AKI in the current study, the occurrence of tachycardia was statistically significant, though not statistically related to mortality. Experimental evidence suggests that hemodynamic changes observed after hemotoxic viper bite can simulate sepsis. The recorded tachycardia in the current study may merely reflect appropriate baroreflex activity to optimize cardiac output and blood pressure. And in the same way as sepsis, persistent tachycardia after adequate volume expansion, may be associated with worsened clinical outcomes. (Kanjanabuch \&Sitprija, 2008; Lanspa et al., 2017).

Hypotension is a key factor favoring the development of AKI due to prerenal insult. A variety of factors contribute to the occurrence of hypotension. Viperidae snake venom increases vascular permeability via the degradation of capillary basement membranes increasing extravasation of plasma from intravascular compartment with decrease in intravascular volume that may compromise circulation. . Additionally it can enhance the release of vasoactive substances, particularly bradykinin, resulting in vasodilatation and myocardial depression which can lead to hypotension without any bleeding manifestations. However in the current study hypotension was statistically insignificant among AKI cases irrespective of mortality, which may reflect the important role of proper evaluation and hemodynamic support of cases with repletion of the fluid deficit and optimisation of intravascular volume (Adukauskienè et al., 2011; Singh et al., 2016; Aye et al., 2017).

Significant increase was found in the occurrence of bleeding manifestations, increased INR and thrombocytopenia among cases with AKI. These findings were similarly recorded in nonsurvivors, in addition to low hemoglobin levels, thus can be viewed as prelude to their mortality. Comparably, Kumar (2012) and Vikrant et al. (2017) noted that hemotoxic viper bite with local and systemic hemorrhagic manifestations indicative of coagulopathy or thrombocytopenia represented the most common clinical features associated with enhanced renal ischemia and development of renal failure.

Snake venom hemotoxins have structural and functional diversity that can result in hemorrhagic, coagulopathic and/or hypotensive effects thereby severely interfering with blood coagulation and platelet function. Biological activities responsible for bleeding tendencies include direct fibrinogenolytic activity resulting in defibrination, which is often exacerbated by venom-induced consumption coagulopathy. Blood clotting disturbances are mediated by the action of procoagulant toxins which activate clotting factors found towards the end of the clotting cascade, such as Factor $\mathrm{X}$ and prothrombin, leading to consumption of the limited amounts of clotting factors physiologically (Isbister et al, 2009; Maduwage \& Isbister, 2014) . Moreover, hemorrhagic components disrupt the integrity of capillaries by proteolytic degradation of key structural components of the basement membrane and extracellular matrix promoting extravasation, rupture and local hemorrhage (Fox \& Serrano, 2005; Casewell et al, 2015; Gutiérrez et al, 2016).

In the current study, thrombocytopenia contributed to venom-induced coagulopathies. This reflected the action of snake venom on platelets. Platelet modulating components include C-type lectins, disintegrins and certain metalloproteinases. These components can either induce platelet aggregation, by Von Willebrand factor or collagenmediated activation, or inhibit their aggregation by potent blockade of integrin receptors found on the surface of platelets. Platelet modulation by inhibition and/or activation leads to platelet depletion (Rucavado et al, 2005; Chakrabarty \& Sarkar, 2016; Kini \& Koh, 2016).

The presence of local manifestations was common among all the cases enrolled in the current study with statistically insignificant relation with both AKI and the associated mortality. The signs and symptoms are consistent with continued local tissue injury and destruction including swelling, blisters, hemorrhage, dermal and muscle necrosis (Sonmez, 2014). 
Local toxicity is the earliest manifestation of viper envenomation, however, its association with AKI and poor outcome was debatable. In agreement to the findings in the current study, Patil et al. (2012) noted that local cellulitis as a common finding, indicates the vasculotoxic nature of envenomation regardless of the outcome. Whilst Mukhopadhyay et al. (2016) and Aye et al. (2017) considered severity as significant adverse predictor and included a grading system of cellulitis. Evidence based guidelines of treatment emphasized that because snake envenomation is a dynamic disease state, grading assigned at a single point in time may be a poor representation of overall severity

Local clinical effects often occur as the result of several venom components especially metalloproteinase, serine proteinases, phospholipases A2, nucleotidases, and hyaluronidases. Pathophysiological scenario of local tissue injury is characterized by degradation of extracellular matrix and basement membrane structure in capillary blood vessels, skeletal muscle fibers and dermal-epidermal junctions. As a result, blistering and hemorrhage occurs, in addition to myonecrosis with affection of muscle regenerative process leading to fibrosis and permanent tissue loss after snakebites. The damaged extracellular matrix favors diffusion of venom toxins, leading to both systemic complications and local tissue damage. Moreover, prominent local inflammatory response occurs due to several factors which further complicates venom-induced dermatonecrosis. These factors include: acute tissue damage, presence of protein fragments of diverse biological activities, release of inflammatory mediators, and activation of endogenous proteinases derived from resident tissue cells or invading leukocytes (Gutiérrez \& Rucavado, 2000; Amog et al, 2016; Gutiérrez et al., 2016).

The parenteral administration of animalderived anti-venoms constitutes the mainstay in the therapy of snakebite envenoming (World Health organization, 2010). Venom-specific immunoglobulin $\mathrm{G}$ fab fragment works by binding and neutralizing venom toxins, facilitating their redistribution away from target tissues and their elimination from the body. Although the total dose of antivenom required may vary widely, aggressive dosing strategy exceeding typical dosing may be needed in severe envenomation. The higher doses employed and the longer duration of hospital stay for cases complicated with AKI and subsequent mortality in the current study reflect the degree of severity which necessitated both rapid control and prolonged follow up for eminent complications and the possible recurrence (Lavonas et al., 2011; Gouda et al., 2017).

Collectively, findings in the current study support findings by previous authors; AKI after envenomation by vipers is due to the interplay of different mechanisms. Important implicated events include: alteration of renal hemodynamics glomerular and enhanced renal ischemia due to hypofiltration by bleeding, depletion of intravascular volume by extravasation, microthrombi formation by consumption coagulopathy. Direct nephrotoxicity by venom constituents, immunologic reaction and pigmenturia may contribute to the development of AKI after viper snake bite (Kanjanabuch \& Sitprija, 2008; Kumar, 2012; Al-Johany et al., 2015).

Hemotoxic events following viper envenomations are related to morbidity and mortality in cases of renal failure and should be expected after envenomation by vipers with a serious course of intoxication with greater susceptibility with increasing age and delayed time to antivenom therapy.

\section{Recommendations}

Acute kidney injury is a potential complication of viper envenomation in Egypt, however its nonrecognition can be complicated by poor outcome. Support materials for health-care providers at all levels of the health service could help to disseminate appropriate scientific knowledge .Being undoubtedly multifactorial, evaluation of clinical manifestations of envenomation and the renal status of viper bite victims both at baseline and during hospital stay is recommended: to allow identification of AKI patients, enable monitoring of AKI severity, and predict patient outcome.

Community education, taking medical care of snake bite closer to those at risk, and efficient supply-chain for antivenom are important to ensure effective timely management thus reducing morbidity and mortality in viper envenomed cases.

Further research is needed to assess more accurate local estimates of incidence and mortality, using health center surveys - both retrospective and prospective - in combination to household surveys .

\section{References}

Adukauskienė D, Varanauskienè E and Adukauskaitè A (2011): Venomous snakebites.Medicina (Kaunas). 47:461-7.

Al-Johany AM, Al-Sadoon MK, Moneim AEA et al., (2015): Histological, molecular and biochemical detection of renal injury after Echis pyramidum snake envenomation in rats. Saudi journal of biological sciences, 22(3):302-311.

Amog PU, Yariswamy M, Joshi V et al., (2016): Local tissue damage induced by Echis carinatus venom: neutralization by Albizia Lebbeck seed aqueous extract in mice model. J Pharm Res. 10(4):167-175. 
Athappan G, Balaji MV, Navaneethan U et al., (2008): Acute renal failure in snake envenomation: A large prospective study. Saudi J Kidney Dis Transpl. 19:404 -10.

Aye KP, Thanachartwet V, Soe C et al., (2017): Clinical and laboratory parameters associated with acute kidney injury in patients with snakebite envenomation: a prospective observational study from Myanmar. BMC nephrology, 18 (1):92110.

Baha EDS (2006): A guide to the reptiles and amphibians of Egypt. Cairo and New York: The American University in Cairo Press, Ch 25.pp. 303-316.

Bellomo R, Kellum JA and Ronco C (2012): Acute kidney injury. The Lancet, 380(9843): 756-766.

Bernardoni JL, Sousa LF, Wermelinger LS et al., (2014): Functional variability of snake venom metalloproteinases: adaptive advantages in targeting different prey and implications for human envenomation. PLoS One, 9(10):e109651.

Casewell N, Sunagar K, Takacs Z et al., (2015): Snake venom metalloprotease enzymes. In: Venomous Reptiles \& Their Toxins: Evolution, Pathophysiology \& Biodiscovery, Fry B (ed.), Oxford University Press, New York, Ch 23. pp. 347-363.

Chakrabarty D and Sarkar A (2016): Cytotoxic Effects of Snake Venoms. In Snake venoms, Gopalakrishnakone $\mathrm{P}$, Inagaki $\mathrm{H}$, Vogel CW et al., (Eds.) Springer Netherlands, Ch 14. pp. 327-342.

Dharod MV, Patil TB, Deshpande AS et al., (2013): Clinical predictors of acute kidney injury following snake bite envenomation. North American Journal of Medical Sciences, 5(10): 594-599.

Fox JW and Serrano SMT (2005): Structural considerations of the snake venom metalloproteinases, key members of the M12 reprolysin family of metalloproteinases. Toxicon, 45: 969985.

Gouda AS, Elnabarawy NA and Badawy SM (2017): A study of snakebite envenomation cases admitted to Egyptian national poisoning center. Acta Medica International, 4(2):34-40.

Gutiérrez JM and Rucavado A (2000). Snake venom metalloproteinases: their role in the pathogenesis of local tissue damage. Biochimie, 82(9-10):841-850.

Gutiérrez JM, Escalante T, Rucavado A et al., (2016): A comprehensive view of the structural and functional alterations of extracellular matrix by snake venom metalloproteinases (SVMPs): novel perspectives on the pathophysiology of envenoming. Toxins, 8 (10):304-324.

Harshavardhan L, Lokesh AJ, Tejeshwari HL, et al., (2013): A study on the acute kidney injury in snake bite victims in a tertiary care centre. J Clin Diagn Res. 7:853-6.

Isbister GK, Duffull SB, Brown SG et al., (2009): Failure of antivenom to improve recovery in Australian snakebite coagulopathy. QJM. 102:563-8.

Kanjanabuch T and Sitprija V (2008): Snakebite nephrotoxicity in Asia. Semin nephrol. 28:363-72.

Kasturiratne A, Wickremasinghe AR, de Silva N et al., (2008): The global burden of snakebite: a literature analysis and modelling based on regional estimates of envenoming and deaths. PLoS medicine, 5(11): e218.

Kenney W, Wilmore J \& Costill D, (2015): Cardiorespiratory responses to acute exercise. In: Physiology of Sport and Exercise, Tocco A, Maurer K \& Walsh K., (eds.), $6^{\text {th }}$ ed., Champaign, Human Kinetics, USA, Ch 8. pp. 195-222.

Kini RM and Koh CY (2016): Metalloproteases affecting blood coagulation, fibrinolysis and platelet aggregation from snake venoms: definition and nomenclature of interaction sites. Toxins, 8(10):284-311.

Kotchen T A (2015). Hypertensive Vascular Disease. In: Harrison's principles of internal medicine, Kasper D, Fauci A, Hauser S, et al.,(eds.), $19^{\text {th }}$ ed., e-Book, McGraw- Hill Education Medical , New York, Ch. 10.

Kumar V (2012): Special Section: Renal Diseases Snake Bite Induced Acute Kidney Injury Health Sciences, 1(2):JS007.

Lanspa MJ, Shahul S, Hersh A et al.,(2017): Associations among left ventricular systolic function, tachycardia, and cardiac preload in septic patients. Annals of intensive care, 7(1):17.

Lavonas EJ, Ruha AM, Banner W et al., (2011): Unified treatment algorithm for the management of crotaline snakebite in the United States: results of an evidenceinformed consensus workshop. BMC emergency medicine, 11(1):2-17.

Lopes JA and Jorge S (2013): The RIFLE and AKIN classifications for acute kidney injury: a critical and comprehensive review. Clinical Kidney Journal, 6(1):814.

Maduwage K and Isbister GK (2014): Current treatment for venom-induced consumption 
coagulopathy resulting from snakebite. PLoS neglected tropical diseases, 8 (10):e3220.

Manafa PO, Osmond EO, Onyenekwe CC et al., (2016): Assessment of Tumour Necrosis Factor-Alpha (Tnf-A) and Creatinine Levels In Echis Ocellatus Bite Victims in Jos Metropolis, Nigeria. European Scientific Journal, 12(21):70-80.

Mandal L (2015): Venomous Snake Bite. PostGraduate Medical Journal of NAMS, 12 (01):57-65.

Mukhopadhyay P, Mishra R, Mukherjee D et al., (2016): Snakebite mediated acute kidney injury, prognostic predictors, oxidative and carbonyl stress: A prospective study. Indian J Nephrol .26:427-33.

Osmani AH, Durrani R and Ara J (2007): Morbidity resulting from delayed presentation of snake bites cases. J Surg Pak .12:31-33.

Patil TB, Bansod YV and Patil MB (2012): Snake bite induced acute renal failure: a study of clinical profile and predictors of poor outcome. World Journal of Nephrology and Urology, 1(2-3):59-65.

Potdukhe P, Naitam B, Gandhi P et al., (2017): A Study of Acute Kidney Injury in Patients with Snakebite Envenomation. JMSCR, 5 (4): 19854-70.

Rucavado A, Soto M, Escalante T et al., (2005) Thrombocytopenia and platelet hypoaggregation induced by Bothrops asper snake venom: toxins involved and their contribution to metalloproteinase induced pulmonary hemorrhage. Thrombosis and Haemostasis, 94:123131.
Singh RR, Uraiya D, Kumar A et al., (2016): Early demographic and clinical predictors of developing acute kidney injury in snake bite patients: A retrospective controlled study from an Indian tertiary care hospital in North Eastern Uttar Pradesh India. Indian J Crit Care Med. 20:404-8.

Sonmez B (2014): Clinical Aspects and Emergent Management of Snake Bites Presented to Emergency Department. Journal of Clinical and Analytical Medicine, 6(101):558-561.

VACSERA - Egyptian Holding Company for Biological Products \& Vaccines (2002): VACSERA Info: Venom Antiserum Polyvalent (Equine) Lyophilized. Available from: http://egyvac.vacsera.com/egyvacProfile.p $\mathrm{df}$

Vikrant S, Jaryal A and Parashar A (2017): Clinicopathological spectrum of snake bite-induced acute kidney injury from India. World journal of nephrology, 6(3):150-161.

Weinstein JR and Anderson S (2010): The aging kidney: Physiological changes. Advances in Chronic Kidney Disease, 17(4): 302307.

World Health Organization (2010): Guidelines of prevention and clinical management of snakebite in Africa Regional Office of Africa Brazzaville-Republic of Congowww.aho.afro.who.int/

World Health Organization (2016): Guidelines for the management of snake-bites, 2nd edition. South East Asia Regional Office www.who.int $>$... > Regional Publications > SEARO Publications . 


\section{الملخص العربي \\ المعايير السريرية والمعملية المرتبطة بالإصابة الكلوية الحادة في حالات لدغ الحيات \\ هدى محمد صلاح الدين عبد العزيز و رباب نبيل حافظا}

تتنتشر فصائل الحيات في جميع أنحاء مصروترتبط العضات السامة بالمظاهرالموضعية والجهازية، والتى يمكن أن تتفاقم بحدوث

إصابة كلوية حادة.

الغرض من الدراسة : تقييم الإصابة الكلوية الحادة في حالات التسمم من الحيات المنتشرة محليا فيما يتعلق بمعدل الحدوث و

المعايير السريرية والمعملية والنتيجة النهائية .

الطرق : أجريت الدراسة الحالية على جميع المرضى، من الجنسين، الذين تم حجزهم في مركز علاج التسمم بمستشفيات جامعة

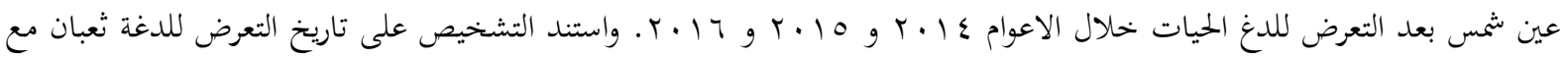

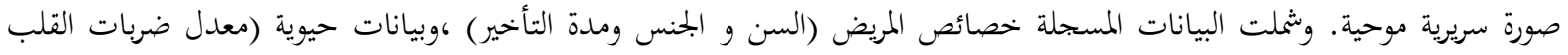

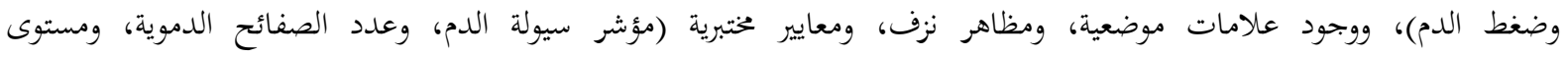

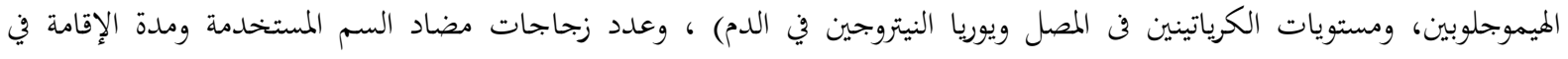

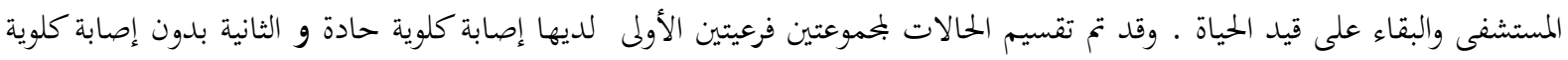
حادة.

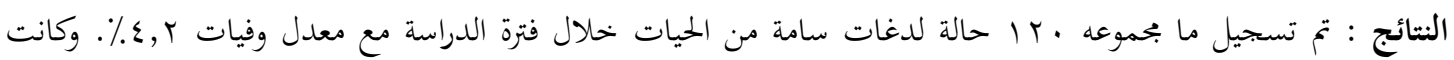

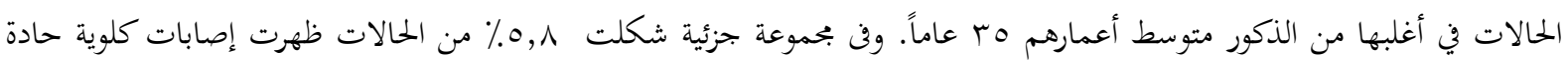

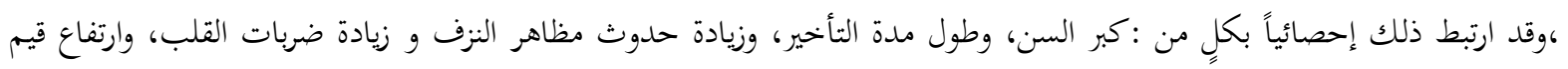

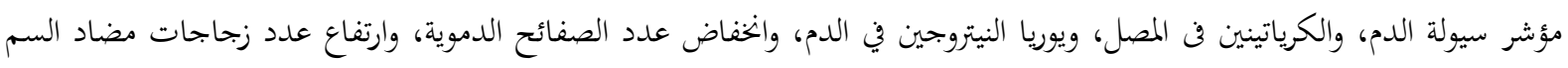

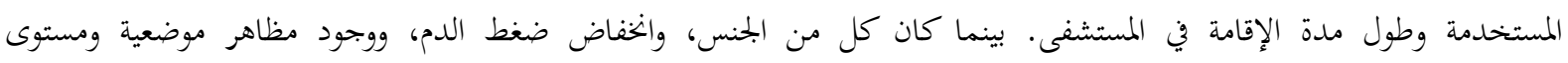
الهيموجلوبين غير ذوى دلالة إحصائية بين حالات الإصابة الكلوية الحادة.

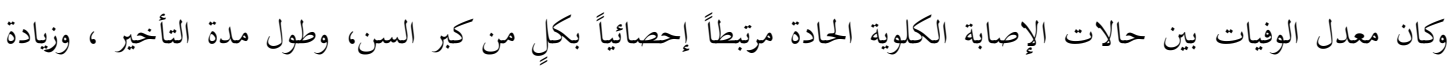

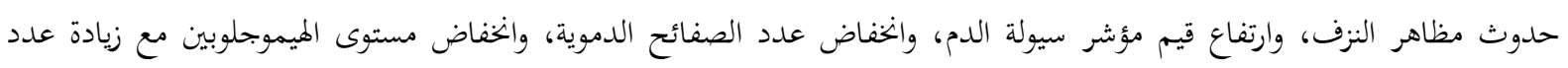

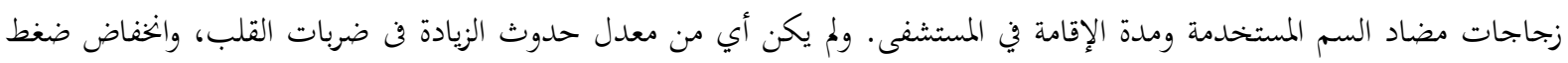

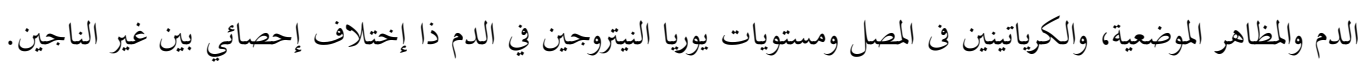

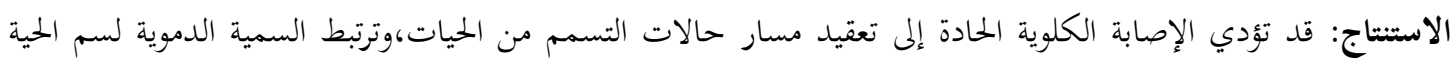

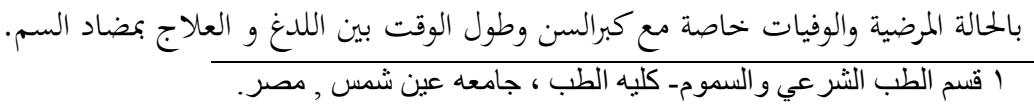

